\title{
Development of a clinical practice guideline for assessment, prevention and treatment of pain in children with cancer
}

Erik A.H. Loeffen ${ }^{1}$, A. Font-Gonzalez², Renée L. Mulder², L. Lee Dupuis ${ }^{3,4}$, M.D. van de Wetering², L.C.M. Kremer $^{2}$, F. Campbell ${ }^{5}$, W.J.E. Tissing ${ }^{1}$, on behalf of the Pain in Children with Cancer Guideline Working Group

\begin{tabular}{|l|}
\hline \multicolumn{1}{|c|}{ Introduction } \\
\hline $\begin{array}{l}\text { High-quality evidence-based guidelines for supportive } \\
\text { care in children with cancer are needed. Pain in children } \\
\text { with cancer has been identified as an area where many } \\
\text { clinicians seek guidance. }\end{array}$ \\
\hline
\end{tabular}

\section{Objectives}

To develop a clinical practice guideline (CPG) for pain

in children with cancer with recommendations on

1. assessment of pain;

2. pharmacological treatment of
a) tumor-related pain
b) treatment-related pain;
c) procedure-related pain;

3. physical and psychological treatment

a) tumor- and treatment-related pain

b) procedure-related pain.

\section{Materials and Methods}

The international and inter-professional guideline panel comprises a core group and six working groups and includes $\mathbf{4 4}$ individuals. Previously 25 clinical questions were prioritized to be answered through systematic literature reviews. After performing the literature appraisal and independent dual data extraction of the included studies (see figure 1), the project group met in Amsterdam (35/44 members attending) to complete and discuss the evidence-to-decision frameworks and formulate draft recommendations.

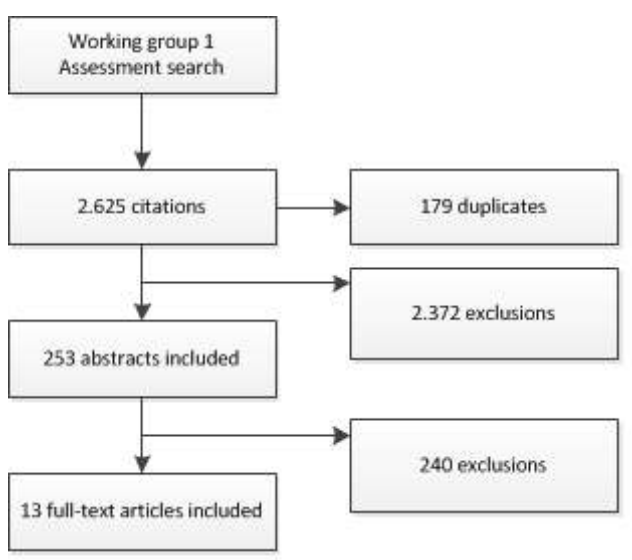

Image 1. Current status of the project.

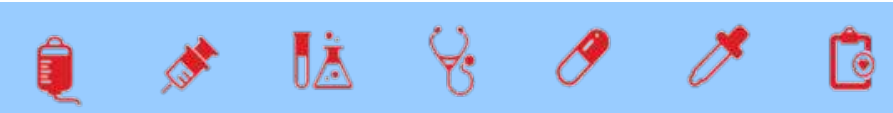

AFFILIATIONS of Pediatric Oncology/Hematology, UMCG, Groningen, NL Department of Pediatric Oncology, AMC, Amsterdam, NL ${ }^{3}$ Department of Pharmacy and Research Institute, SickKids, Toronto, CAN ${ }^{4}$ Leslie Dan Faculty of Pharmacy, University of Toronto, Toronto, CAN ${ }^{5}$ Department of Anesthesia and Pain Medicine, SickKids, Toronto, CAN

ACKNOWLEDGEMENTS guidelines for supportive care in The project "Towards evidence-based git childhood oncology" is supported by the Alpe dHuZes foundalion/Dutch Cancer Society (RUG 2013-6345). We thank the Dutch pediatric oncology patient and parent association "Vereniging Ouders, Kinderen en Kanker"

\begin{tabular}{|l|}
\hline \multicolumn{1}{|c|}{ Want to connect? } \\
\hline$\square \quad$ eah.loeffen@umcg.nl \\
$\qquad \quad$ @erikloeffen \\
\hline
\end{tabular}

\section{Results}

Various draft recommendations were formulated for all working groups. These recommendations are currently being discussed with the members not present at the guideline meeting and various patient- and parentrepresentatives. Hereafter the recommendations will be finalized.

\begin{tabular}{|lcr|}
\hline \multicolumn{3}{|c|}{ Conclusion } \\
\hline We have developed a CPG \\
regarding pain in children with \\
cancer. & Currently & the
\end{tabular}
recommendations are being finalized, thereafter the guideline will be written in three parts. In the next phase we will work on implementation of the CPG.

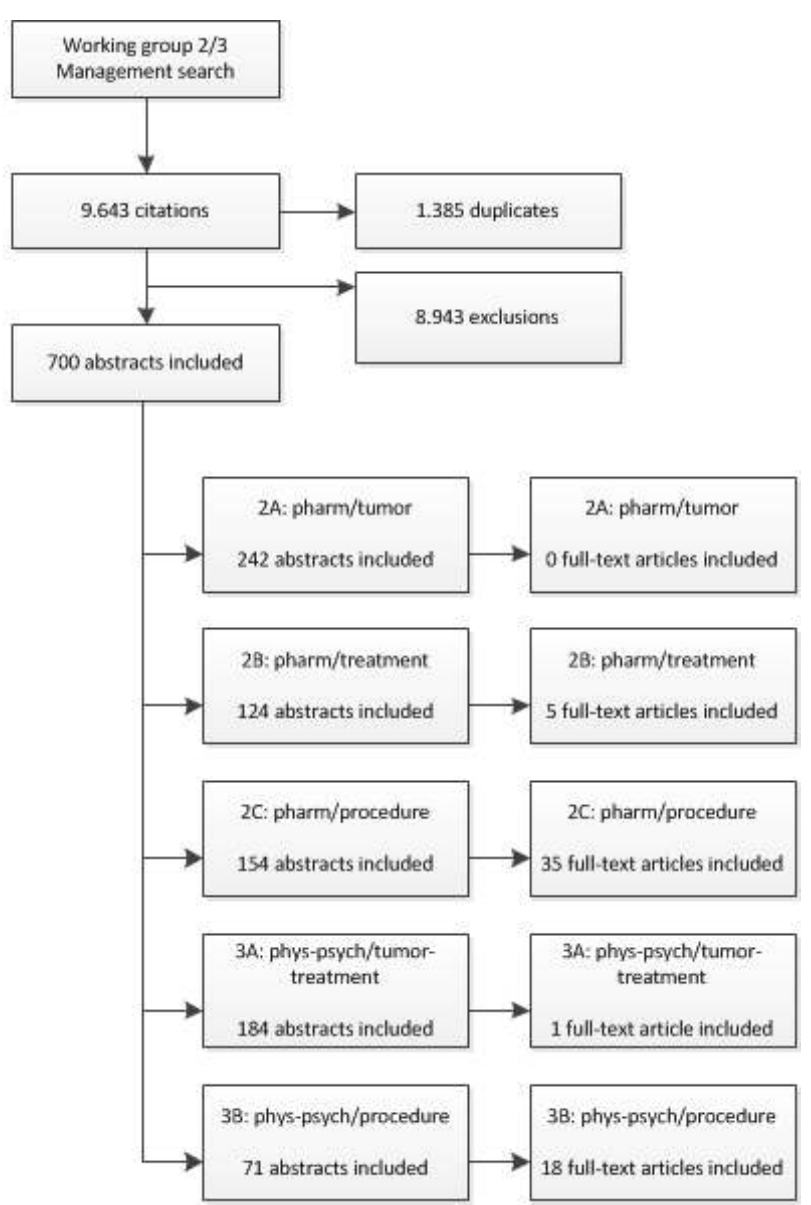

